\title{
Projective Unitary Antiunitary Representations of Locally Compact Groups
}

\author{
K. R. Parthasarathy \\ Statistical Laboratory, University of Manchester
}

Received August 25, 1969

\begin{abstract}
We give here a systematic presentation of the theory of projective representations when antiunitary operators are present. In particular the imprimitivity theorem of Mackey is proved in this situation and all the unitary antiunitary representations of the extended Poincaré group are derived.
\end{abstract}

\section{§ 1. Introduction}

In the mathematical formulation of quantum mechanics proposed by von Neumann (cf. $[3,8,9]$ ) the set of all propositions concerning a quantum mechanical system is an orthocomplemented lattice. The simplest example of such a lattice is the lattice $\mathscr{L}(\mathscr{H})$ of all closed subspaces of a separable Hilbert space $\mathscr{H}$. The observables in such a system turn out to be self adjoint operators in $\mathscr{H}$. In order to construct the standard observables like energy, linear, angular and spin angular momenta etc., in such a system it is necessary to study the effect of coordinate transformations by a group $G$ of symmetries. In this context the representations of $G$ in the group $\mathscr{A}(\mathscr{H})$ of automorphisms of the lattice $\mathscr{L}(\mathscr{H})$ is of great importance.

By the extension of a theorem of Wigner (cf. [8], Theorem 7.27, page 167) it is known that every automorphism $\tau$ of $\mathscr{L}(\mathscr{H})$ is induced by a unitary or antiunitary operator $U^{\tau}$ on $\mathscr{H}$. The operator $U^{\tau}$ is determined uniquely only up to a scalar multiple of modulus unity. Suppose $\mathscr{U}(\mathscr{H})$ is the group of all unitary and antiunitary operators on $\mathscr{H}$ and $\mathscr{I}(\mathscr{H})$ is the normal subgroup consisting of scalar multiples of identity. Then Wigner's theorem can be reformulated as follows: the group $\mathscr{A}(\mathscr{H})$ is isomorphic with the quotient group $\mathscr{U}(\mathscr{H}) / \mathscr{I}(\mathscr{H})$. We shall denote this quotient by $P U A(\mathscr{H})$ and call it the projective unitary antiunitary group of $\mathscr{H} . \mathscr{U}(\mathscr{H})$ with the weak (operator) topology (which is equivalent to the strong topology) is a complete and separable metric group in which $\mathscr{I}(\mathscr{H})$ is a compact subgroup. Thus the quotient topology in $P U A(\mathscr{H})$ can be carried over to $\mathscr{A}(\mathscr{H})$ in order to make it a topological group. 
In this paper we are interested in studying the continuous representations of $G$ in the group $P U A(\mathscr{H})$ or equivalently $\mathscr{A}(\mathscr{H})$. Even though there is a very detailed account of projective unitary representations in the papers of Mackey [2, 4]; Wigner [10] and others, there does not seem to exist a systematic theory of projective unitary antiunitary or $P U A$ representations. Some examples of such representations in the case of the Poincare group (or the inhomogeneous full Lorentz group) may be found in the works of Foldy [1] and Streater and Wightman [7]. We shall extend the results of Mackey on transitive imprimitivity systems when $P U A$ representations are considered and derive as an application all the "positive mass" PUA representations of the Poincaré group. This answers some of the questions raised in Foldy [1].

\section{§ 2. The $P U A$ Group of a Hilbert Space}

In this section we shall study some of the basic properties of unitary and antiunitary operators, describe the projective unitary antiunitary group and finally prove a result concerning its topology and Borel structure.

Definition 2.1. Let $\mathscr{H}_{1}$ and $\mathscr{H}_{2}$ be two complex separable Hilbert spaces with inner products $(\cdot, \cdot)_{1}$ and $(\cdot, \cdot)_{2}$ respectively. A map $V: \mathscr{H}_{1} \rightarrow \mathscr{H}_{2}$ is called antiunitary if $V$ is one one, onto and satisfies the following properties:

(1) $V(x+y)=V x+V y$ for all $x, y \in \mathscr{H}_{1}$,

(2) $V a x=\bar{a} V x$ for all $x \in \mathscr{H}_{1}$ and scalars $a$,

(3) $(V x, V y)_{2}=(y, x)_{1}$ for all $x, y \in \mathscr{H}_{1}$.

If $\mathscr{H}_{1}=\mathscr{H}_{2}=\mathscr{H}, V$ is called an antiunitary operator on $\mathscr{H}$.

From now onwards let $\mathscr{H}$ stand for a fixed complex separable Hilbert space with inner product $(\cdot, \cdot)$. We shall denote by $\mathscr{U}^{+}(\mathscr{H})$ and $\mathscr{U}^{-}(\mathscr{H})$ respectively the set of all unitary operators and the set of all antiunitary operators on $\mathscr{H}$. The set $\mathscr{U}(\mathscr{H})=\mathscr{U}^{+}(\mathscr{H}) \cup \mathscr{U}^{-}(\mathscr{H})$ is a group under the composition operation. This is called the unitary antiunitary group or simply the UA group of $\mathscr{H}$. If there is no ambiguity we shall drop the $\mathscr{H}$ within brackets and denote these by $\mathscr{U}, \mathscr{U}^{+}$, and $\mathscr{U}^{-}$. It is obvious that $\mathscr{U}^{+}$ is a normal subgroup of $\mathscr{U}$ and

$$
\begin{aligned}
& \mathscr{U}^{+} \mathscr{U}^{-}=\mathscr{U}^{-} \mathscr{U}^{+}=\mathscr{U}^{-} ; \\
& \mathscr{U}^{-} \mathscr{U}^{-}=\mathscr{U}^{+} .
\end{aligned}
$$

If $V \in \mathscr{U}^{-}$, then $V \mathscr{U}^{+}=\mathscr{U}^{+} V=\mathscr{U}^{-}$. In particular, if $V$ is any fixed antiunitary operator then every antiunitary operator $W$ can be written as 
$U V$ where $U$ is a unitary operator. Thus the group $\mathscr{U} / \mathscr{U}^{+}$has only two elements.

Lemma 2.1. Let $(X, \mathscr{S})$ be a Borel space and $P$ and $Q$ be two projection valued measures on $\mathscr{S}$. Suppose there exists a $W \in \mathscr{U}^{-}$such that

$$
W P(E) W^{-1}=Q(E) \text { for all } E \in \mathscr{S} .
$$

Then there exists a $U \in \mathscr{U}^{+}$such that

$$
U P(E) U^{-1}=Q(E) \text { for all } E .
$$

Proof. By the Hahn-Hellinger theorem we may, without loss of generality, assume that $\mathscr{H}$ is of the form $\bigoplus_{r} L_{2}\left(\mu_{r}\right)$ where $\mu_{r}$ is a sequence of measures on $\mathscr{S}$ and $P(E)$ is just multiplication by $\chi_{E}$ where $\chi_{E}$ is the indicator function of $E$. Consider the map $V_{0}: f \rightarrow \bar{f}$ where bar stands for complex conjugation and $\boldsymbol{f}$ is an element of $\mathscr{H}$. $V_{0}$ is an antiunitary operator which commutes with all the $P(E)$. Thus the operator $U=W V_{0}$ is unitary and satisfies the required property. This completes the proof.

In the above lemma we used the fact that complex conjugation is an antiunitary operator. We note that it is an antiunitary operator whose square is identity. The next lemma asserts that every such antiunitary operator is unitarily equivalent to a complex conjugation.

Lemma 2.2. Let $V_{1}$ and $V_{2}$ be antiunitary operators such that $V_{1}^{2}=V_{2}^{2}=I$. Then there exists a $U \in \mathscr{U}^{+}$such that $U V_{1} U^{-1}=V_{2}$.

Proof. Consider the unitary operator $W=V_{2} V_{1}$. Suppose

$$
W=\int_{0}^{2 \pi} e^{i \lambda} P(d \lambda)
$$

where $P$ is a projection valued measure in $[0,2 \pi]$. Let

$$
U=\int_{0}^{2 \pi} e^{i \lambda / 2} P(d \lambda)
$$

We have

$$
I=V_{2}^{2}=W V_{1} W V_{1} .
$$

Thus $W^{-1}=V_{1} W V_{1}$. This implies

$$
\begin{aligned}
\int_{0}^{2 \pi} e^{-i \lambda} P(d \lambda) & =V_{1} \int_{0}^{2 \pi} e^{i \lambda} P(d \lambda) V_{1} \\
& =\int_{0}^{2 \pi} e^{-i \lambda} V_{1} P(d \lambda) V_{1} .
\end{aligned}
$$


Hence $V_{1} P(E) V_{1}=P(E)$ for all Borel sets $E \subset[0,2 \pi]$. Thus

$$
\begin{aligned}
V_{1} U V_{1} & =V_{1} \int_{0}^{2 \pi} e^{i \lambda / 2} P(d \lambda) V_{1} \\
& =\int_{0}^{2 \pi} e^{-i \lambda / 2} V_{1} P(d \lambda) V_{1} \\
& =\int_{0}^{2 \pi} e^{-i \lambda / 2} P(d \lambda) \\
& =U^{-1} .
\end{aligned}
$$

Now we have

$$
U V_{1} U^{-1}=U V_{1}\left(V_{1} U V_{1}\right)=W V_{1}=V_{2}
$$

Since $U$ is unitary, this completes the proof of the lemma.

We shall now assign the $U A$ group $\mathscr{U}$ the weak topology. A sequence $U_{n}$ converges to $U$ in $\mathscr{U}$ if and only if, for every pair $x, y \in \mathscr{H},\left(U_{n} x, y\right)$ $\rightarrow(U x, y)$ as $n \rightarrow \infty$. It is easy to prove that this topology is equivalent to the strong topology. Thus $U_{n} \rightarrow U$ in $\mathscr{U}$ if and only if, for every $x \in \mathscr{H}$, $\left\|U_{n} x-U x\right\| \rightarrow 0$ as $n \rightarrow \infty$. Introduce the metric

$$
d(U, V)=\sum_{i=1}^{\infty} \frac{\left\|U x_{i}-V x_{i}\right\|}{2^{i}} \text { for } U, V \in \mathscr{U}
$$

where $x_{1}, x_{2}, \ldots$ is any fixed orthonormal basis in $\mathscr{H}$. This is a left invariant metric in $\mathscr{U}$ which induces the required topology in $\mathscr{U}$ and makes it a complete and separable metric group.

Let $\mathscr{I} \subset \mathscr{U}$ be the subset $\{c I,|c|=1\}$. Then $\mathscr{I}$ is a compact normal subgroup of $\mathscr{U}$. The quotient group $\mathscr{U} / \mathscr{I}$ is called the projective unitary antiunitary group or simply the PUA group of $\mathscr{H}$. We shall denote the $P U A$ group by $\tilde{\mathscr{U}}$ and the canonical homomorphism from $\mathscr{U}$ onto $\tilde{\mathscr{U}}$ by $\sim$. Thus $\sim$ map sends a unitary or antiunitary operator $U$ to the coset $\tilde{U}=U \mathscr{I}$ in $\tilde{\mathscr{U}}$. Endowed with the quotient topology, $\tilde{\mathscr{U}}$ becomes a separable metric group. The next lemma implies that $\tilde{\mathscr{U}}$ is actually a complete and separable metric group.

Lemma 2.3. If $G$ is a complete and separable metric group and $H \subset G$ is a closed subgroup, then the quotient space $G / H$ of left cosets admits a metric which induces the quotient topology and makes $\mathrm{G} / \mathrm{H}$ a complete and separable metric space.

Proof ${ }^{1}$. We may assume that the metric $d$ in $G$ is right invariant. Let

$$
N_{k}=\left\{x: d(g, e)<2^{-k}\right\}, \quad k=1,2 \ldots
$$

\footnotetext{
1 This proof was suggested to the author by A. Tortrat.
} 
be a decreasing sequence of symmetric open neighbourhoods of the identity $e$.

Let $\dot{g}=\pi g$ be the coset $g H$ for every $g \in G . \pi$ is the canonical map from $G$ onto $G / H$ which takes the point $g$ to the coset containing $g$. Define

$$
d^{0}\left(\dot{g}_{1}, \dot{g}_{2}\right)=\inf _{h \in H} d\left(g_{1}, g_{2} h\right) .
$$

It is easy to verify that $d^{0}$ defines a metric in $G / H$ and induces the quotient topology. Now let $\dot{g}_{n}$ be a sequence of points in $G / H$ such that

$$
\lim _{n, m \rightarrow \infty} d^{0}\left(\dot{g}_{n}, \dot{g}_{m}\right)=0 \text {. }
$$

This implies that for every $k$, there exists an integer $n_{k}$ such that $\left\{n_{k}\right\}$ is monotonic increasing and

$$
\pi^{-1} g_{n} \subset N_{k} \pi^{-1} g_{m} \text { for all } n, m \geqq n_{k} .
$$

Choose a sequence $g_{k}^{\prime} \in \pi^{-1} g_{n_{k}}$ as follows: $g_{1}^{\prime}$ is any element in $\pi^{-1} g_{n_{1}}$. By (2.2) $\pi^{-1} g_{n} \cap N_{1} g_{1}^{\prime} \neq \theta$ for all $n \geqq n_{1}$. Hence choose $g_{2}^{\prime} \in \pi^{-1} g_{n_{2}} \cap N_{1} g_{1}^{\prime}$. Then $\pi^{-1} g_{n} \cap N_{2} g_{2}^{\prime} \neq \theta$ for all $n \geqq n_{2}$. Choose $g_{3}^{\prime} \in \pi^{-1} g_{n_{3}} \cap N_{2} g_{2}^{\prime}$. Repeat this procedure.

It is clear that

$$
g_{l}^{\prime} \in N_{l-1} N_{l-2} \ldots N_{k} g_{k}^{\prime} \text { for all } l>k .
$$

By (2.1) and the triangle inequality we have

$$
N_{l-1} N_{l-2} \ldots N_{k} \subset N_{k-1} \text {. }
$$

Hence $g_{l}^{\prime} \in N_{k-1} g_{k}^{\prime}$ for all $l>k$. Since $G$ is complete under the metric $d$ it follows that $g_{k}^{\prime} \rightarrow g \in G$ as $k \rightarrow \infty$. Thus $\dot{g}_{k}^{\prime} \rightarrow \dot{g}$ in $G / H$. Since $\dot{g}_{k}^{\prime}=\dot{g}_{n_{k}}$, it follows that $\dot{g}_{n} \rightarrow \dot{g}$ as $n \rightarrow \infty$. Thus every Cauchy sequence converges in $G / H$. This completes the proof.

Corollary 2.1. The PUA group $\tilde{\mathscr{U}}$ of a complex separable Hilbert space is a complete and separable metric group. In particular, $\tilde{\mathscr{U}}$ with its Borel structure derived from its topology is a standard Borel space.

Corollary 2.2. There exists a one one Borel map $\eta$ from $\tilde{\mathscr{U}}$ into $\mathscr{U}$ such that $\eta\left(U^{Y^{2}}{ }^{\sim}=U^{\sim}\right.$.

Proof. Since $\mathscr{U}$ and $\mathscr{U}^{\sim}$ are complete and separable metric spaces and the map $U \rightarrow U^{\sim}$ is continuous, the existence of the map $\eta$ follows from a theorem of Kuratowski (cf. [5], Theorem 3.9, page 21).

Remark. Since $\sim$ is an open map and $\mathscr{U}^{+}$and $\mathscr{U}^{-}$are both open closed subsets of $\mathscr{U}$, it follows that their images $\tilde{U}^{+}$and $\tilde{\mathscr{U}}^{-}$are disjoint both open closed subsets of $\tilde{\mathscr{U}}$. $\tilde{\mathscr{U}}^{+}$is a normal subgroup of $\tilde{\mathscr{U}}$. Further

$$
\tilde{\mathscr{U}}=\tilde{\mathscr{U}}^{+} \cup \tilde{\mathscr{U}}^{-} ; \tilde{\mathscr{U}}^{+} \tilde{\mathscr{U}}^{-}=\tilde{\mathscr{U}}^{-} \tilde{\mathscr{U}}^{+}=\tilde{\mathscr{U}}^{-} ; \tilde{\mathscr{U}}^{-} \tilde{\mathscr{U}}^{-}=\tilde{\mathscr{U}}^{+} \text {. }
$$




\section{§3. PUA Representations}

Throughout this section let $G$ denote a locally compact second countable group and $\tilde{\mathscr{U}}$ be the $P U A$ group of a complex separable Hilbert space $\mathscr{H}$. We give $G$ its natural Borel structure and $\tilde{\mathscr{U}}$ the topology described in $\S 2$.

Definition 3.1. A Borel homomorphism from $G$ into $\tilde{\mathscr{U}}$ is called a projective unitary antiunitary representation or simply a $P U A$ representation of $G$ in $\mathscr{H}$.

Lemma 3.1. Any $P U A$ representation of $G$ is continuous. For a $P U A$ representation $g \rightarrow U_{g}^{\tilde{g}}$ of $G$, the set $G^{+}=\left\{g: U_{g}^{\tilde{g}} \in \tilde{\mathscr{U}}^{+}\right\}$is a both open closed normal subgroup of $G$ such that $G / G^{+}$has at the most two elements. If $G^{-}=\left\{g: U_{g}^{\sim} \in \tilde{\mathscr{U}}^{-}\right\}$, then $G^{+}$and $G^{-}$are the cosets which constitute the group $G / G^{+}$.

Proof. By Corollary 2.1 to Lemma 2.3, $\tilde{\mathscr{U}}$ is a separable metric group. Hence by a well known result of Mackey (cf. [6], Theorem 2.2, page 10), the map $g \rightarrow U_{g}^{\sim}$ is continuous. By the Remark at the end of $\S 2, \widetilde{\mathscr{U}}^{+}$is a both open closed normal subgroup of $\tilde{\mathscr{U}}$. Since $G^{+}$is the inverse image of $\tilde{\mathscr{U}}^{+}$through a continuous map, it is both open closed. The rest of the properties are quite straightforward to prove.

Corollary 3.1. If in a PU A representation $g \rightarrow U_{g}^{\sim}$ of $G$, there exists a $g_{0}$ such that $U_{g_{0}}^{\sim} \in \tilde{\mathscr{U}}^{-}$, then $G$ cannot be connected.

Definition 3.2. The decomposition of $G$ into $G^{+}$and $G^{-}$occurring in Lemma 3.1 is called the $U A$ decomposition of $G$ associated with the $P U A$ representation $g \rightarrow U_{g}^{\sim}$.

We shall now investigate how a $P U A$ representation can be lifted to a "multiplier representation" in the $U A$ group $\mathscr{U}$. Suppose $g \rightarrow U_{g}^{\sim}$ is a $P U A$ representation of $G$. Making use of the cross section map $\eta$ of Corollary 2.2 to Lemma 2.3 we can construct a measurable map $g \rightarrow \eta\left(U_{g}^{\sim}\right)$ from $G$ into $\mathscr{U}$. Since $\eta\left(U_{g}^{\sim}\right)^{\sim}=U_{g}^{\sim}$, we may, without loss of generality, assume that $U_{g}=\eta\left(U_{g}\right)$. Then $g \rightarrow U_{g}$ is a measurable map and for any two elements $g_{1}, g_{2} \in G,\left(U_{g_{1}} U_{g_{2}}\right)^{\sim}=\tilde{U_{g_{1} g_{2}}}$. Hence there exists a complex number $\sigma\left(g_{1}, g_{2}\right)$ of modulus unity such that

$$
U_{g_{1}} U_{g_{2}}=\sigma\left(g_{1}, g_{2}\right) U_{g_{1} g_{2}} \text { for all } g_{1}, g_{2} \in G .
$$

We may always take $U_{e}=I$ where $e$ is the identity element of $G$. Then

$$
\sigma(e, g)=\sigma(g, e)=1 \text { for all } g \in G .
$$

Suppose that $G=G^{+} \cup G^{-}$is the associated $U A$ decomposition of the $P U A$ representation $g \rightarrow U_{g}$. Computing $U_{g_{1}} U_{g_{2}} U_{g_{3}}$ in two different ways 
as $U_{g_{1}}\left(U_{g_{2}} U_{g_{3}}\right)$ and $\left(U_{g_{1}} U_{g_{2}}\right) U_{g_{3}}$, we obtain

and

$$
\begin{aligned}
& U_{g_{1}}\left(U_{g_{2}} U_{g_{3}}\right)=\sigma\left(g_{1}, g_{2} g_{3}\right) \sigma\left(g_{2}, g_{3}\right) U_{g_{1} g_{2} g_{3}} \quad \text { if } \quad g_{1} \in G^{+}, \\
& =\sigma\left(g_{1}, g_{2} g_{3}\right) \bar{\sigma}\left(g_{2}, g_{3}\right) U_{g_{1} g_{2} g_{3}} \text { if } g_{1} \in G^{-}
\end{aligned}
$$

$$
\left(U_{g_{1}} U_{g_{2}}\right) U_{g_{3}}=\sigma\left(g_{1}, g_{2}\right) \sigma\left(g_{1} g_{2}, g_{3}\right) U_{g_{1} g_{2} g_{3}} \text { for all } g_{1}, g_{2}, g_{3} \in G .
$$

Thus $\sigma$ satisfies the following equation:

$$
\left.\begin{array}{rlll}
\sigma\left(g_{1}, g_{2}\right) \sigma\left(g_{1} g_{2}, g_{3}\right) & =\sigma\left(g_{1}, g_{2} g_{3}\right) \sigma\left(g_{2}, g_{3}\right) & \text { if } & g_{1} \in G^{+}, \\
& =\sigma\left(g_{1}, g_{2} g_{3}\right) \bar{\sigma}\left(g_{2}, g_{3}\right) & \text { if } & g_{1} \in G^{-}
\end{array}\right\}
$$

Considering the properties of $\sigma$ in (3.2) and (3.3), we introduce the following definition.

Definition 3.3. Suppose $G^{+}$is a both open closed normal subgroup of $G$ such that the group $G / G^{+}$has at the most two cosets $G^{+}$and $G^{-}$(where $G^{-}$can be empty). A Borel function $\sigma$ defined on $G \times G$ and taking values on the unit circle of the complex plane is called a multiplier with respect to $G^{+}$if it satisfies Eqs. (3.2) and (3.3). A Borel map $g \rightarrow U_{g}$ from $G$ into $\mathscr{U}$ is called a multiplier representation if there exists a multipler $\sigma$ such that Eq. (3.1) is satisfied. In this case it is also called a $\sigma$ representation.

Thus our previous discussion can be summed up in the form of a theorem.

Theorem 3.1. Let $G$ be a locally compact second countable group and $g \rightarrow U_{g}$ be a $P U A$ representation of $G$. Then there exists a multiplier representation $g \rightarrow V_{g}$ of $G$ such that $V_{g}^{\sim}=U_{g}^{\sim}$ for all $g \in G$. Conversely every multiplier representation $g \rightarrow V_{g}$ of $G$ determines a $P U A$ representation $g \rightarrow V_{g}$ of $G$.

Definition 3.4. Let $g \rightarrow U_{g}^{\sim}$ be a $P U A$ representation of $G$. Any multiplier representation $g \rightarrow V_{g}$ of $G$ such that $V_{g}^{\sim}=U_{g}^{\sim}$ for all $g \in G$ is called a version of the given $P U A$ representation.

Remark. Suppose now that $g \rightarrow V_{g}$ and $g \rightarrow W_{g}$ are two versions of a $P U A$ representation with multipliers $\sigma$ and $\sigma^{\prime}$. Since $V_{g}^{\sim}=W_{g}^{\sim}$ for all $g \in G$, there exists a Borel function $a(g)$ on $G$ such that $|a(g)|=1$ and $W_{g}=a(g) V_{g}$ for all $g \in G$. Hence $\sigma$ and $\sigma^{\prime}$ satisfy the following identity:

$$
\begin{aligned}
\sigma^{\prime}\left(g_{1}, g_{2}\right) & =\frac{a\left(g_{1}\right) a\left(g_{2}\right)}{a\left(g_{1} g_{2}\right)} \sigma\left(g_{1}, g_{2}\right) \quad \text { if } \quad g_{1} \in G^{+}, \\
& =\frac{a\left(g_{1}\right) \overline{a\left(g_{2}\right)}}{a\left(g_{1} g_{2}\right)} \sigma\left(g_{1}, g_{2}\right) \text { if } \quad g_{1} \in G^{-}
\end{aligned}
$$

where $G=G^{+} \cup G^{-}$is the associated $U A$ decomposition. 
Definition 3.5. A multiplier representation $g \rightarrow U_{g}$ of $G$ is said to be irreducible if there exists no non trivial proper closed subspace invariant under all the $U_{g}, g \in G$, A $P U A$ representation is said to be irreducible if it has an irreducible version.

Two multiplier representations $g \rightarrow U_{g}^{(i)}, i=1,2$ in Hilbert spaces $\mathscr{H}_{i}, i=1,2$ respectively are said to be equivalent if there exists a unitary or antiunitary operator $U$ from $\mathscr{H}_{1}$ onto $\mathscr{H}_{2}$ such that $U U_{g}^{(1)} U^{-1}=U_{g}^{(2)}$ for all $g \in G$.

Two PUA representations are said to be equivalent if they have equivalent versions.

Remark. Two equivalent $P U A$ representations of $G$ have the same associated $U A$ decomposition for the group $G$. If the equivalence is effected by a unitary operator, then they have versions with the same multiplier. If it is effected by an antiunitary operator, then they have versions whose multipliers $\sigma_{1}$ and $\sigma_{2}$ satisfy the equation $\sigma_{2}=\bar{\sigma}_{1}$.

Because of the Remark after Definition 3.4 and the comments made above we shall introduce the following definition.

Definition 3.6. Two multipliers $\sigma$ and $\sigma^{\prime}$ are said to be trivially equivalent if they are defined with respect to the same normal subgroup $G^{+}$ and there exists a Borel function $a(g)$ on $G$ such that $|a(g)|=1$ and Eq. (3.4) is satisfied. $\sigma$ and $\sigma^{\prime}$ are said to be equivalent if either $\sigma$ and $\sigma^{\prime}$ are trivially equivalent or $\bar{\sigma}$ and $\sigma^{\prime}$ are trivially equivalent.

\section{§ 4. Imprimitivity Systems}

Let $G$ be a locally compact second countable group and $H \subset G$ be a closed subgroup. We shall denote by $X=G / H$ the homogeneous space of left cosets in which $G$ acts transitively through left translation. Let $x$ denote an arbitrary point of $X$ and $x_{0}$ the point corresponding to the coset $H$. We shall denote by $\pi$ the map from $G$ onto $X$ which takes the element $g$ to the coset $g H$. The space $X$ with the quotient topology becomes a locally compact second countable space. Let $\mathscr{B}_{X}$ be the Borel $\sigma$-field of $X$.

Following Mackey we shall define the notion of an "imprimitivity system".

Definition 4.1. Let $\mathscr{H}$ be a complex separable Hilbert space. By an imprimitivity system for $G$ on $X$, we mean a pair consisting of a multiplier representation $g \rightarrow U_{g}$ of the group $G$ and a projection valued measure $E \rightarrow P(E), E \in \mathscr{B}_{X}$ in $\mathscr{H}$ such that

$$
U_{g} P(E) U_{g}^{-1}=P(g E) \text { for all } g \in G, E \in \mathscr{B}_{X} .
$$

We shall denote this system by $\left\{\mathscr{H}, U_{g}, P(E)\right\}$. 
Remark. It is clear that the above definition is meaningful if $X$ is replaced by any Borel space where $G$ acts as a group of Borel automorphisms. We shall, however, confine our attention to the transitive case since other "ergodic $G$ actions" have not been completely understood even in the unitary case. In this connection the reader is referred to Mackey [4].

Definition 4.2. An imprimitivity system $\left\{\mathscr{H}, U_{g}, P(E)\right\}$ for the group $G$ on the homogeneous space $X$ is said to be irreducible if there is no proper non zero subspace which is invariant under all the operators $U_{g}$ and $P(E), g \in G, E \in \mathscr{B}_{X}$.

Two imprimitivity systems $\left\{\mathscr{H}^{i}, U_{g}^{(i)}, P^{(i)}(E)\right\}, i=1,2$ for the group $G$ on the homogeneous space $X$ are said to be equivalent if there exists a unitary or antiunitary map $V$ from $\mathscr{H}^{1}$ onto $\mathscr{H}^{2}$ such that

$$
\begin{array}{lll}
\left(V U_{g}^{(1)} V^{-1}\right)^{\sim}=U_{g}^{(2)} \sim & \text { for all } & g \in G, \\
V P^{(1)}(E) V^{-1}=P^{(2)}(E) & \text { for all } & E \in \mathscr{B}_{X} .
\end{array}
$$

We shall now study the problem of classifying the imprimitivity systems of $G$ up to equivalence. More or less we follow Mackey and use his results in many places. However, there are some differences and these will become clear as we proceed.

By a quasi invariant measure on $\left(X, \mathscr{B}_{X}\right)$ we mean a non zero $\sigma$-finite measure $\mu$ on $\mathscr{B}_{X}$ such that $\mu(E)=0$ if and only if $\mu(g E)=0$ for all $g \in G, E \in \mathscr{B}_{X}$. It is well known that quasi invariant measures exist on $X$ and any two quasi invariant measures are equivalent in the sense of measure theory. We shall choose and fix a quasi invariant measure $\mu$ throughout our discussion in this section.

For every finite or countable cardinal $n, L_{2}(\mu, n)$ will stand for the Hilbert space which is the direct sum of $n$ copies of the Hilbert space $L_{2}(\mu)$ of complex square integrable functions. Let $\mathbb{C}^{n}$ denote the $n$ dimensional complex Hilbert space if $n<\infty$ and the Hilbert space of square summable sequences of complex numbers, i.e.,

$$
\left\{\boldsymbol{a}: \boldsymbol{a}=\left(a_{1}, a_{2}, \ldots\right): \sum_{i=1}^{\infty}\left|a_{i}\right|^{2}<\infty\right\} \text { if } n=\infty \text {. }
$$

We shall denote an arbitrary element of $\mathfrak{C}^{n}$ by $\boldsymbol{a}$ and the inner product in $\mathfrak{C}^{n}$ by $\langle.,$.$\rangle . The inner product in L_{2}(\mu, n)$ will be denoted by $(.,$.$) . Any$ element of $L_{2}(\mu, n)$ can be written as $\boldsymbol{f}(x)=\left(f_{1}(x), f_{2}(x), \ldots\right)$ where $\int \sum_{i}\left|f_{i}(x)\right|^{2} d \mu(x)<\infty$. The space $L_{2}(\mu, n)$ admits a canonical projection valued measure $P^{0}(E), E \in \mathscr{B}_{X}$ defined by

$$
P^{0}(E) \boldsymbol{f}=\left(\chi_{E} f_{1}, \chi_{E} f_{2}, \ldots\right)
$$


for all $f=\left(f_{1}, f_{2}, \ldots\right) \in L_{2}(\mu, n)$ where $\chi_{E}$ is the indicator function of the set $E$.

We shall now prove a series of lemmas before proceeding to the statement of the main results.

Lemma 4.1. Let $\left\{\mathscr{H}, U_{g}, P(E)\right\}$ be an imprimitivity system for $G$ on $X$. Then there exists an equivalent imprimitivity system $\left\{L_{2}(\mu, n), V_{g}, P^{0}(E)\right\}$ where $\mu$ is a quasi invariant measure on $X, n$ is a finite or countable cardinal and $P^{0}(E)$ is the canonical projection valued measure.

Proof. Definition 4.1 implies that the projection valued measures $P(E)$ and $P(g E), E \in \mathscr{B}_{X}$ are equivalent through the unitary or antiunitary operator $U_{g}$. Hence by Lemma 2.1, they are unitarily equivalent. Now an application of the Hahn-Hellinger theorem and the argument of Mackey [2] yield the proof of the lemma.

In the space $L_{2}(\mu, n)$, the complex conjugation which maps $\boldsymbol{f}$ to $\overline{\boldsymbol{f}}$ is a canonical antiunitary operator. By the discussion in $\S 2$, it follows that every antiunitary operator is the product of a unitary operator and this conjugation. Making use of this fact and following the arguments of Mackey [2] one can prove the following lemma.

Lemma 4.2. Let $\left\{L_{2}(\mu, n), V_{g}, P^{0}(E)\right\}$ be an imprimitivity system for $G$ on $X$. Let $G=G^{+} \cup G^{-}$be the $U A$ decomposition of $G$ associated with the $P U$ A representation $g \rightarrow V_{g}$. Then there exist functions $C(g, x)$ and $D(g, x)$ defined respectively on $G^{+} \times X$ and $G^{-} \times X$ and taking values in the space of unitary operators in $\mathfrak{E}^{n}$ such that

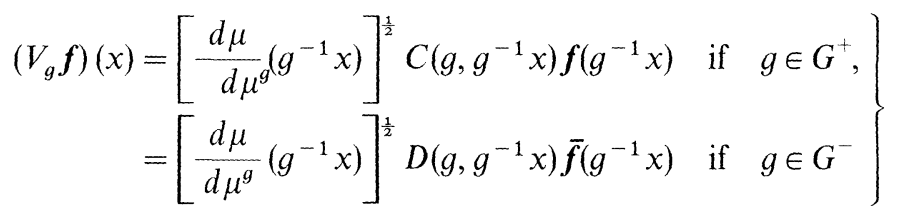

where $\mu^{g}$ is the quasi invariant measure defined by the equation $\mu^{g}(E)$ $=\mu(g E), E \in \mathscr{B}_{X}$.

If $G^{-}$is empty then we have only a unitary imprimitivity system and this case has been studied in great detail by Mackey [2]. Hence we shall concentrate our attention on the case when $G^{-} \neq \theta$. In such a situation two different types of imprimitivity systems arise according as the action of the subgroup $G^{+}$on $X$ is transitive or not.

a) The Case when $G^{+}$Action in $X$ is Transitive and $G^{-} \neq 0$

Suppose $\left\{L_{2}(\mu, n), V_{g}, P^{0}(E)\right\}$ is an imprimitivity such that the subgroup $G^{+}=\left\{g: V_{g}\right.$ is unitary $\}$ acts transitively on $X$. Since $G^{+} G^{-}=G^{-}$, this implies that the stability subgroup $H$ of the point $x_{0}$ has non empty 
intersection with $G^{-}$. Let

$$
H^{+}=H \cap G^{+}, \quad H^{-}=H \cap G^{-} .
$$

Then by Lemma 3.1, $H^{+}$is a both open closed normal subgroup of $H$ such that $H / \mathrm{H}^{+}$consists of two cosets $\mathrm{H}^{+}$and $\mathrm{H}^{-}$. Choose and fix a point $h_{0} \in H^{-}$. Then $G^{-}=G^{+} h_{0}, H^{-}=H^{+} h_{0}$. The element $h_{0}$ induces an automorphism of the group $G^{+}$through the map $g \rightarrow h_{0} g h_{0}^{-1}$. We shall write $h_{0}[g]$ for $h_{0} g h_{0}^{-1} . H^{+}$is invariant under this automorphism. Further $h_{0}^{2} \in H^{+}$. The map $\pi$ from $G$ onto $X$ is continuous and in particular measurable. Hence by a result of Kuratowski (cf. [5], Theorem 3.9, page 21) we can construct a one one measurable map $\gamma$ from $X$ into $G$ such that $\pi \gamma$ is the identity map. Thus $\gamma(x) x_{0}=x$ for all $x . \gamma$ is called a Borel cross section map for $\pi$. We shall choose and fix such a cross section map throughout our discussion.

Lemma 4.3. Let $\left\{L_{2}(\mu, n), V_{g}, P^{0}(E)\right\}$ be an imprimitivity system and let $\sigma$ be the multiplier of the representation $g \rightarrow V_{g}$. Then there exists an equivalent imprimitivity system with the same $L_{2}(\mu, n)$ and the canonical projection valued measure $P^{0}$ such that the function $C(g, x)$ of Lemma 4.2 is given by the formula

$$
C(g, x)=\frac{\sigma(g, \gamma(x))}{\sigma\left(\gamma(g x), \gamma(g x)^{-1} g \gamma(x)\right)} L_{\gamma(g x)^{-1} g \gamma(x)} \quad \text { if } \quad g \in G^{+}
$$

where $h \rightarrow L_{h}$ is a $\sigma$ unitary representation of the subgroup $H^{+}$.

Proof. For the subgroup $G^{+}$and its transitive action in the space $X$, the pair consisting of the $\sigma$ representation $g \rightarrow V_{g}$ and the projection valued measure $E \rightarrow P^{0}(E)$ is a transitive imprimitivity system in the sense of Mackey. Every $V_{g}, g \in G^{+}$is unitary. The first equation in (4.1) and the Eq. (4.2) simply express the fact that the $\sigma$ representation $g \rightarrow V_{g}$ of $G^{+}$ is induced by the $\sigma$-representation $h \rightarrow L_{h}$ of the subgroup $H^{+}$. Hence Lemma 4.3 is just a restatement of Mackey's result [2]. This completes the proof.

From now onwards we shall tacitly assume that $C(g, x)$ is defined by (4.2). We shall choose the orthonormal basis $\boldsymbol{v}_{i}=(0,0, \ldots, 0,1,0, \ldots)$ where 1 is written at the $i$ th position in the vector for $i=1,2, \ldots$ In this basis we shall construct the matrices of the operators $C(g, x)$ and $D(g, x)$ in the Hilbert space $\mathbb{C}^{n}$. Let us denote these matrices by the same symbols. Thus $C(g, x)$ and $D(g, x)$ will be $n \times n$ unitary matrices. For any matrix $A$, let $\bar{A}$ be the matrix obtained by taking the complex conjugate of every entry.

By writing a.e.x. we shall refer with respect to the quasi invariant measure $\mu$ on $X$. "Almost everywhere" statements in a group will always be relative to Haar measure. With these conventions we have the following lemma. 
Lemma 4.4. Let $\left\{L_{2}(\mu, n), V_{g}, P^{0}(E)\right\}$ be an imprimitivity system and let $\sigma$ be the multiplier of $g \rightarrow V_{g}$. Suppose $V_{g}$ is defined by (4.1) and $C(g, x)$ is defined according to (4.2). Then the matrix valued functions $C(g, x)$ and $D(g, x)$ satisfy the following equations:

$$
\begin{array}{r}
C\left(g_{1}, g_{2} x\right) C\left(g_{2}, x\right)=\sigma\left(g_{1}, g_{2}\right) C\left(g_{1} g_{2}, x\right) \\
\quad \text { for all } x \in X, g_{1}, g_{2} \in G^{+}, \\
\begin{array}{r}
D\left(g_{1}, g_{2} x\right) \overline{D\left(g_{2}, x\right)}=\sigma\left(g_{1}, g_{2}\right) C\left(g_{1} g_{2}, x\right) \\
\text { a.e.x. for every } g_{1}, g_{2} \in G^{-}, \\
C\left(g_{1}, g_{2} x\right) D\left(g_{2}, x\right)=\sigma\left(g_{1}, g_{2}\right) D\left(g_{1} g_{2}, x\right) \\
\text { a.e.x. for every } g_{1} \in G^{+}, g_{2} \in G^{-}, \\
D\left(g_{1}, g_{2} x\right) \overline{C\left(g_{2}, x\right)}=\sigma\left(g_{1}, g_{2}\right) D\left(g_{1} g_{2}, x\right) \\
\text { a.e.x. for every } g_{1} \in G^{-}, g_{2} \in G^{+} .
\end{array}
\end{array}
$$

Proof. The first equation follows by direct verification. The last three equations can be deduced easily from (4.1) and the equation $V_{g_{1}} V_{g_{2}}$ $=\sigma\left(g_{1}, g_{2}\right) V_{g_{1} g_{2}}$.

Lemma 4.5. Under the same conditions as in Lemma 4.4, for every $g \in G^{+}$, the function $D\left(g h_{0}, x\right)$ is given by the equation

where

$$
D\left(g h_{0}, x\right)=\bar{\sigma}\left(g, h_{0}\right) C\left(g, h_{0} x\right) D(x) \text { a.e.x. }
$$

$$
D(x)=a(x) L_{\gamma\left(h_{0} x\right)^{-1} h_{0}[\gamma(x)]} \Gamma, \text { a.e. } x .
$$

$\Gamma$ is a constant unitary matrix with the property

$$
\begin{gathered}
L_{h_{0}[h]}=\alpha(h) \Gamma \bar{L}_{h} \Gamma^{-1} \quad \text { for all } h \in H^{+}, \\
\Gamma \bar{\Gamma}=c L_{h_{0}^{2}} .
\end{gathered}
$$

and $a(x)$ and $\alpha(h)$ are scalar functions of modulus unity.

Proof. Put $g_{2}=h_{0}, g_{1}=g \in G^{+}$in (4.5). Then we get (4.7) with $D(x)=D\left(h_{0}, x\right)$. For any matrix valued function $A(x)$ defined on $X$, let $A^{0}(g)=A(\pi(g))$. If $A$ is measurable on $X$, then $A^{0}$ is measurable on $G$. Putting $g_{1}=h_{0}, g_{2}=g \in G^{+}$in (4.6) we obtain

$$
D(g x)=\sigma\left(h_{0}, g\right) D\left(h_{0}[g] h_{0}, x\right) \overline{C(g, x)}^{-1} \text { a.e.x. for every } g \in G^{+} .
$$

By "lifting" both sides of this equation to the group $G$ and using Fubini's theorem we obtain

$$
\begin{array}{r}
\left.D^{0}\left(g_{1} g_{2}\right)=\sigma\left(h_{0}, g_{1}\right) \bar{\sigma}\left(h_{0}\left[g_{1}\right], h_{0}\right) C^{0}\left(h_{0}\left[g_{1}\right], h_{0} g_{2}\right) D^{0}\left(g_{2}\right) \overline{C^{0}\left(g_{1}, g_{2}\right.}\right)^{-1} \\
\text { a.e. } g_{1} \in G^{+}, g_{2} \in G .
\end{array}
$$


Let $g_{2} \in G^{+}$be a fixed point such that the above equation holds a.e. $g_{1} \in G^{+}$. Let $D^{0}\left(g_{2}\right)=\Gamma_{1}$ at that point. Then

$$
D^{0}(g)=a_{1}(g) C^{0}\left(h_{0}\left[g g_{2}^{-1}\right], h_{0} g_{2}\right) \Gamma_{1} \overline{C^{0}\left(g g_{2}^{-1}, g_{2}\right)^{-1}} \quad \text { a.e. } g \in G^{+}
$$

where $a_{1}(g)$ is scalar. By (4.3) we conclude the existence of another constant matrix $\Gamma_{2}$ and a scalar function $a_{2}(g)$ such that

$$
D^{0}(g)=a_{2}(g) C^{0}\left(h_{0}[g], h_{0}\right) \Gamma_{2}{\overline{C^{0}(g, e)^{-1}}}^{-1} \text { a.e. } g \in G^{+} .
$$

Since $D^{0}(g h)=D^{0}(g)$ for every $h \in H^{+}$, we obtain from (4.2),

$$
L_{h_{0}[h]} \Gamma_{2} \bar{L}_{h}^{-1}=\alpha(h) \Gamma_{2}, \quad h \in H^{+}
$$

for some scalar $\alpha(h)$. Putting $g_{1}=g_{2}=h_{0}$ in (4.4), and using (4.2), we have

$$
\Gamma_{2} \bar{\Gamma}_{2}=c L_{h_{0^{2}}}
$$

where $c$ is a scalar of modulus unity. Writing $\Gamma_{2}=\Gamma$ and putting $g=\gamma(x)$ in (4.11) we obtain the required result.

Lemma 4.6. In the Hilbert space $\mathbb{C}^{n}$, define the operators $M_{h}, h \in H$ as follows:

$$
\begin{aligned}
M_{h} \boldsymbol{a} & =L_{h} \boldsymbol{a} \text { if } h \in H^{+}, \boldsymbol{a} \in \mathbb{C}^{n} \\
M_{h_{0}} \boldsymbol{a} & =\overline{\sigma\left(h, h_{0}\right)} L_{h} \Gamma \overline{\boldsymbol{a}} \text { if } h h_{0} \in H^{-}, \boldsymbol{a} \in \mathbb{V}^{n}
\end{aligned}
$$

where $L_{h}, h \in H^{+}$and $\Gamma$ are as in Lemma 4.5. Then the map $h \rightarrow M_{h}$ is a multiplier representation of the group $H$ whose multiplier $\tilde{\sigma}$ is given by

$$
\begin{aligned}
& \tilde{\sigma}\left(h_{1}, h_{2}\right)=\sigma\left(h_{1}, h_{2}\right) \quad \text { if } \quad h_{1} \in H^{+}, h_{2} \in H \\
& =\chi\left(h_{2}\right) \quad \text { if } \quad h_{1} \in H^{-}, h_{2} \in H
\end{aligned}
$$

where $\chi$ is a character on $H$.

Proof. That $h \rightarrow M_{h}$ defines a multiplier representation of $H$ is an immediate consequence of Eqs. (4.9) and (4.10) of Lemma 4.5. Since $M_{h}=L_{h}$ for $h \in H^{+}$, it is clear that $\tilde{\sigma}=\sigma$ on $H^{+} \times H^{+}$. If $h_{1} \in H^{+}$and $h_{2}=h h_{0}, h \in H^{+}$, then for any $\boldsymbol{a} \in \mathbb{C}^{n}$,

$$
\begin{aligned}
M_{h_{1}} M_{h h_{0}} \boldsymbol{a} & =L_{h_{1}} \bar{\sigma}\left(h, h_{0}\right) L_{h} \Gamma \overline{\boldsymbol{a}} \\
& =\bar{\sigma}\left(h, h_{0}\right) \sigma\left(h_{1}, h\right) L_{h_{1} h} \Gamma \overline{\boldsymbol{a}} \\
& =\bar{\sigma}\left(h, h_{0}\right) \sigma\left(h_{1}, h\right) \sigma\left(h_{1} h, h_{0}\right) M_{h_{1} h h_{0}} \boldsymbol{a} \\
& =\sigma\left(h_{1}, h h_{0}\right) M_{h_{1} h h_{0}} \boldsymbol{a} .
\end{aligned}
$$

Thus $\tilde{\sigma}\left(h_{1}, h h_{0}\right)=\sigma\left(h_{1}, h h_{0}\right)$. Since $\tilde{\sigma}$ and $\sigma$ are multipliers with respect to the same normal subgroup $H^{+} \mathrm{C} H$, it follows that $\tilde{\sigma} \bar{\sigma}$ is also a multiplier for $H$ with respect to $H^{+}$. Let $\phi=\tilde{\sigma} \bar{\sigma}$ on $H \times H$. We have $\phi\left(h_{1}, h_{2}\right)=1$ if $h_{1}, h_{2} \in H^{+}$. A straightforward computation now shows that $\phi\left(h_{1} h_{0}, h_{2}\right)$ 
$=\phi\left(h_{0}, h_{2}\right)$ for all $h_{1} \in H^{+}$and $\phi\left(h_{0}, h h^{\prime}\right)=\phi\left(h_{0}, h\right) \phi\left(h_{0}, h^{\prime}\right)$ for all $h, h^{\prime} \in H$. This completes the proof of the lemma.

Now we are ready to state and prove the first main theorem of this section.

Theorem 4.1. Let $G$ be a locally compact second countable group, $H \subset G$ a closed subgroup and $X=G / H$ the homogeneous space of left cosets. Let $\left\{\mathscr{H}, U_{g}, P(E)\right\}$ be an imprimitivity system for $G$ on $X$. Let $G=G^{+} \cup G^{-}$be the $U A$ decomposition of $G$ with respect to $P U A$ representation $g \rightarrow U_{g}, g \in G$. Suppose that $G^{+}$acts transitively on $X$ and $\sigma$ is the multiplier of the representation $g \rightarrow U_{g}$. Let $\gamma$ be a one one Borel map from $X$ into $G^{+}$such that $\pi \gamma$ is the identity map of $X$ onto itself. Then there exists an equivalent imprimitivity system $\left\{L_{2}(\mu, n), V_{g}, P^{0}(E)\right\}$ where

$$
\begin{aligned}
\left(V_{g} f\right)(x)= & \frac{\sigma\left(g, \gamma\left(g^{-1} x\right)\right)}{\sigma\left(\gamma(x), \gamma(x)^{-1} g \gamma\left(g^{-1} x\right)\right)}\left\{\left(\frac{d \mu}{d \mu^{g}}\right)\left(g^{-1} x\right)\right\}^{\frac{1}{2}} \\
& \cdot M_{\gamma(x)-1{ }^{1} g \gamma\left(g^{-1} x\right)} f\left(g^{-1} x\right),
\end{aligned}
$$

$\mu$ is a quasi invariant measure, $n$ is a finite or countable cardinal, $h \rightarrow M_{h}$ is a $\sigma$ representation of $H$ and $P^{0}$ is the canonical projection valued measure on $\mathscr{B}_{X}$. This imprimitivity system is irreducible if and only if the $\sigma$ representation $h \rightarrow M_{h}$ of $H$ is irreducible.

Proof. Lemmas 4.1-4.3 and 4.5-4.6 imply the existence of an equivalent imprimitivity system $\left\{L_{2}(\mu, n), V_{g}, P^{0}(E)\right\}$ where $V_{g}$ is given by the formula

$$
\begin{aligned}
V_{g} \boldsymbol{f}(x)= & \left\{\frac{d \mu}{d \mu^{g}}\left(g^{-1} x\right)\right\}^{\frac{1}{2}} \frac{\sigma\left(g, \gamma\left(g^{-1} x\right)\right)}{\sigma\left(\gamma(x), \gamma(x)^{-1} g \gamma\left(g^{-1} x\right)\right)} \\
& \cdot \alpha\left(g, g^{-1} x\right) M_{\gamma(x)^{-1} g \gamma\left(g^{-1} x\right)} f\left(g^{-1} x\right)
\end{aligned}
$$

where $\alpha(g, x)$ is a scalar function of modulus unity, $h \rightarrow M_{h}$ is a $\tilde{\sigma}$ representation of $H$ and $\tilde{\sigma}$ is a multiplier of the form given by Lemma 4.6. Further $\alpha(g, x)=1$ if $g \in G^{+}$. Writing the equation $V_{g_{2}} V_{g_{1}}=\sigma\left(g_{2}, g_{1}\right) V_{g_{2} g_{1}}$ for $g_{1}, g_{2} \in G^{-}$and putting $y=g_{2}^{-1} x$, we obtain

$$
\alpha\left(g_{2}, y\right)=\alpha\left(g_{1}, g_{1}^{-1} y\right) \chi\left(\gamma(y)^{-1} g_{1} \gamma\left(g_{1}^{-1} y\right)\right) \text { a.e.y. }
$$

Thus $\alpha(g, y)$ is independent of $g \in G^{-}$. Writing $\alpha(y)$ for $\alpha(g, y)$ we have

$$
\alpha(y) \overline{\alpha\left(g_{1}^{-1} y\right)}=\chi\left(\gamma(y)^{-1} g_{1} \gamma\left(g_{1}^{-1} y\right)\right) \text { a.e.y. }
$$

Writing $\alpha^{0}(g)=\alpha(\pi(g))$, we obtain

$$
\alpha^{0}(g) \overline{\alpha^{0}\left(g_{1}^{-1} g\right)}=\chi\left(\gamma(\pi(g))^{-1} g_{1} \gamma\left(\pi\left(g_{1}^{-1} g\right)\right) \quad\right. \text { a.e.g. }
$$


Putting $g_{1}^{-1} g=g^{\prime}$, and noting that $\chi$ is a homomorphism on $H$, we obtain

Hence

$$
\alpha^{0}(g) \overline{\alpha^{0}\left(g^{\prime}\right)}=\chi\left([\gamma \pi(g)]^{-1} g\right) \overline{\chi\left(\left[\gamma \pi\left(g^{\prime}\right)\right]^{-1} g^{\prime}\right)} .
$$

$$
\alpha^{0}(g)=c \chi\left([\gamma \pi(g)]^{-1} g\right) \quad \text { a.e.g. }
$$

where $c$ is a constant. Since $\alpha^{0}(g h)=\alpha^{0}(g)$ for every $h \in H$, it follows that $\chi(h)=1$ for all $h \in H$ and $\alpha$ is a constant. Without loss of generality we may take this to be unity. Now $h \rightarrow M_{h}$ is indeed a $\sigma$ representation of $H$. This completes the proof of the first part. The irreducibility part is proved exactly as in the unitary case.

Remark. 1. If $H^{-}=H \cap G^{-} \neq 0$, then $M_{h}$ is antiunitary for all $h \in H^{-}$. Further $V_{g}$ is antiunitary for $g \in G^{-}$.

2. Changing the quasi invariant measure $\mu$ yields only an equivalent representation.

3. One may call the representation $g \rightarrow V_{g}$ defined by (4.12) as the $\sigma$ representation induced by $h \rightarrow M_{h}$.

\section{b) The Case when $G^{+}$Action on $X$ is not Transitive and $G^{-} \neq 0$}

In this case the stability subgroup $H$ of the point $x_{0}$ (corresponding to the coset $H$ ) is contained in $G^{+}$. Hence $H^{-}=\theta$. Choose and fix a point $g_{0} \in G^{-}$. The homogeneous space $X$ splits into two orbits $X^{+}$and $X^{-}$ under the action of $G^{+}$where $X^{+}=G^{+} x_{0}, X^{-}=G^{+}\left(g_{0} x_{0}\right)=G^{-} x_{0}$. Let the restrictions of the quasi invariant measure $\mu$ to $X^{+}$and $X^{-}$be $\mu^{+}$and $\mu^{-}$respectively. The Hilbert space $L_{2}(\mu, n)$ splits into a direct sum $L_{2}\left(\mu^{+}, n\right) \oplus L_{2}\left(\mu^{-}, n\right)$. The point $g_{0}$ maps $X^{+}$onto $X^{-}$in a one one manner. For any set $E \subset X$, let $E^{+}=E \cap X^{+}, E^{-}=E \cap X^{-}$.

We can take $\mu^{-}=\mu^{+} g_{0}^{-1}$ without loss of generality. We shall now adopt the matrix notation for an operator in $\mathscr{H}_{1} \oplus \mathscr{H}_{2}$, i.e., an operator $A$ in $\mathscr{H}_{1} \oplus \mathscr{H}_{2}$ will be written as $\left(\begin{array}{ll}A_{11} & A_{12} \\ A_{21} & A_{22}\end{array}\right)$ where $A_{i j}$ is an operator from $\mathscr{H}_{j}$ into $\mathscr{H}_{i}, i, j=1,2$. If $P^{0}, P^{+}$and $P^{-}$are the canonical projection valued measures in $L_{2}(\mu, n), L_{2}\left(\mu^{+}, n\right)$ and $L_{2}\left(\mu^{-}, n\right)$ respectively, we have

$$
P^{0}(E)=\left(\begin{array}{ll}
P^{+}\left(E^{+}\right) & 0 \\
0 & P^{-}\left(E^{-}\right)
\end{array}\right), \quad E \in \mathscr{B}_{X} .
$$

Let $P_{0}$ be the projection valued measure defined with respect to the Hilbert space $L_{2}\left(\mu^{+}, n\right) \oplus L_{2}\left(\mu^{+}, n\right)$ and given by the equation

$$
P_{0}(E)=\left(\begin{array}{ll}
P^{+}\left(E^{+}\right) & 0 \\
0 & P^{+}\left(g_{0} E^{-}\right)
\end{array}\right), \quad E \in \mathscr{B}_{X} .
$$

With these notations we have the following theorem. 
Theorem 4.2. Let $G$ be a locally compact second countable group, $H \subset G$ a closed subgroup and $X=G / H$ the homogeneous space of left cosets. Let $\left\{\mathscr{H}, U_{g}, P(E)\right\}$ be an imprimitivity system for $G$ on $X$. Let $G=G^{+} \cup G^{-}$be the $U A$ decomposition for the PUA representation $g \rightarrow U_{g}^{2}$ of $G$. Suppose that $H \subset G^{+}, G^{-} \neq \theta$ and $g_{0} \in G^{-}$is any fixed point. Let $\sigma$ be the multiplier of $g \rightarrow U_{g}$. Then there exists an equivalent imprimitivity system $\left\{L_{2}\left(\mu^{+}, n\right) \oplus L_{2}\left(\mu^{+}, n\right), V_{g}, P_{0}(E)\right\}$ where $V_{g}$ is given by the equations

$$
\begin{aligned}
V_{g} & =\left(\begin{array}{ll}
V_{g}^{+} & 0 \\
0 & \overline{\alpha(g)} s^{+} V_{g_{0}[g]}^{+} s^{+}
\end{array}\right) \text {if } g \in G^{+} \\
V_{g_{0}} & =\left(\begin{array}{ll}
\frac{0}{\sigma\left(g_{0}, g_{0}\right)} s^{+} V_{g_{0}^{2}}^{+} & 0
\end{array}\right), \\
g_{0}[g] & =g_{0} g g_{0}^{-1}, \quad g \in G^{+}, \\
\alpha(g) & =\sigma\left(g_{0}, g\right) \overline{\sigma\left(g_{0}, g_{0}^{-1}\right)} \sigma\left(g_{0} g, g_{0}^{-1}\right), \quad g \in G^{+},
\end{aligned}
$$

$g \rightarrow V_{g}^{+}$is the unitary $\sigma$ representation in $L_{2}\left(\mu^{+}, n\right)$ for the group $G^{+}$ induced by a unitary $\sigma$ representation $h \rightarrow L_{h}$ of the subgroup $H, s^{+}$denotes the complex conjugation in $L_{2}\left(\mu^{+}, n\right)$ and $P_{0}$ is the projection valued measure defined by (4.13). This imprimitivity system is irreducible if and only if the $\sigma$ representation $h \rightarrow L_{h}$ of $H$ is irreducible.

Proof. Using Lemmas 4.1-4.2 we can replace $\left\{\mathscr{H}, U_{g}, P(E)\right\}$ by $\left\{L_{2}(\mu, n), W_{g}, P^{0}(E)\right\}$ where the $\sigma$ representation $g \rightarrow W_{g}$ is given by the right hand side of (4.1). By the comments made before the statement of the theorem, the Hilbert space $L_{2}(\mu, n)$ splits into a direct sum of two Hilbert spaces $L^{+}=L_{2}\left(\mu^{+}, n\right)$ and $L^{-}=L_{2}\left(\mu^{-}, n\right)$. Since $G^{+}$leaves $X^{+}$ and $X^{-}$invariant it follows from Mackey's theory [2] that $W_{g}$ has the form

$$
W_{g}=\left(\begin{array}{ll}
W_{g}^{+} & 0 \\
0 & W_{g}^{-}
\end{array}\right) \text {if } \quad g \in G^{+}
$$

where $g \rightarrow W_{g}^{ \pm}$in $L^{ \pm}$are two $\sigma$ representations of $G^{+}$, induced from $H$. Since $g_{0}$ maps $X^{+}$onto $X^{-}$and vice versa and $W_{g_{0}}$ is antiunitary, it follows from (4.1) that $W_{g_{0}}$ has the form

$$
W_{g_{0}}=\left(\begin{array}{ll}
0 & B s^{-} \\
A s^{+} & 0
\end{array}\right)
$$

where $s^{+}$and $s^{-}$are complex conjugations in $L^{+}$and $L^{-}$respectively and $A$ and $B$ are unitary maps from $L^{+}$onto $L^{-}$and $L^{-}$onto $L^{+}$respectively. 
Since $W_{g}, g \in G^{+}$and $W_{g_{0}}$ generate a $\sigma$ representation for some multiplier $\sigma$, it follows that

$$
\left.\begin{array}{rl}
W_{g_{0}}^{2} & =\sigma\left(g_{0}, g_{0}\right) W_{g_{0}^{2}} \\
W_{g_{0}} W_{g} W_{g_{0}}^{-1} & =\alpha(g) W_{g_{0}[g]} \text { for all } g \in G^{+}
\end{array}\right\} .
$$

Substituting (4.14) and (4.15) in (4.16) we have

$$
\begin{aligned}
B s^{-} A s^{+} & =\sigma\left(g_{0}, g_{0}\right) W_{g_{0}^{2}}^{+}, \\
\left(B s^{-}\right) W_{g}^{-}\left(B s^{-}\right)^{-1} & =\alpha(g) W_{g_{0}[g]}^{+} .
\end{aligned}
$$

Thus the $\sigma$-representation $g \rightarrow W_{g}^{-}$is "antiunitarily" equivalent to the $\sigma$ representation $g \rightarrow W_{g_{0}[g]}^{+}$. Consider the unitary map from $L^{+} \oplus L^{-}$onto $L^{+} \oplus L^{+}$which sends an element $\left(f, f^{\prime}\right)$ to $\left(f, s^{+} B s^{-} f^{\prime}\right)$. Through this map the $\sigma$ representation $g \rightarrow W_{g}$ of $G$ becomes equivalent to the $\sigma$ representation $g \rightarrow V_{g}$ in $L^{+} \oplus L^{+}$given by

$$
\begin{aligned}
& V_{g}=\left(\begin{array}{ll}
W_{g}^{+} & 0 \\
0 & \overline{\alpha(g)} s^{+} W_{g_{0}[g]}^{+} s^{+}
\end{array}\right) \text {if } g \in G^{+},
\end{aligned}
$$

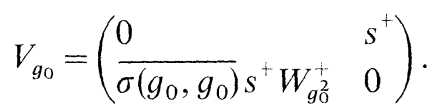

It is easy to verify that (4.17) and (4.18) determine a $\sigma$ representation. Under this equivalence the canonical projection valued measure $P^{0}$ in $L^{+} \oplus L^{-}$is taken into the projection valued measure $P_{0}$ in $L^{+} \oplus L^{+}$defined by (4.13). Writing $V$ instead of $W$ in (4.17) and (4.18) we complete the proof of the first part.

In order to prove the second part we first observe that if the $\sigma$ representation $h \rightarrow L_{h}$ of $H$ is a direct sum of two $\sigma$ representations, then the imprimitivity system described in the statement of the theorem decomposes into a direct sum of two systems. Thus, if $L$ is reducible then the corresponding imprimitivity system is reducible. To prove the converse, let us assume that $L$ is irreducible. Let

$$
K=\left(\begin{array}{ll}
K_{11} & K_{12} \\
K_{21} & K_{22}
\end{array}\right)
$$

be an operator in $L^{+} \oplus L^{+}$which commutes with all $V_{g}, g \in G$ and $P_{0}(E)$, $E \in B_{X}$. The commutativity of $K$ and $P_{0}(E)$ for all $E \in \mathscr{B}_{X}$ implies that $K_{12}=K_{21}=0 . K_{11}$ and $K_{22}$ commute with all $P^{+}(E), E \subset X^{+}$. Further $K_{11}$ and $K_{22}$ commute with all $W_{g}, g \in G^{+}$. Hence from Mackey's imprimitivity theorem it follows that $K_{11}=a I, K_{22}=b I$ where $a$ and $b$ are scalars. The matrix $K=\left(\begin{array}{cc}a I & 0 \\ 0 & b I\end{array}\right)$ commutes with $W_{g_{0}}$. This implies that 
$b=\bar{a}$. Thus the only projection operator which commutes with all $W_{g}, g \in G$ and $P_{0}(E), E \in \mathscr{B}_{X}$ is either 0 or $I$. This completes the proof of the theorem.

\section{$\S 5$. The PU A Representations of the Extended Poincaré Group}

Following Mackey [2] and using the results of $\S 4$ one can construct the irreducible $P U A$ representations of any locally compact second countable group which is a "regular semidirect product" of a connected abelian group and a group of its automorphisms. We shall do this in the special case when the group under consideration is the semidirect product of the four dimensional real Euclidean group $\mathfrak{R}^{4}$ and the group of its automorphisms generated by $S L(2, \mathfrak{C})$, the space reflection and the time reflection. We shall call this the extended Poincare group.

We shall now describe the extended Poincare group in slightly greater detail. In order to do this we need the notion of a semidirect product.

Definition 5.1. Let $N$ and $H$ be locally compact second countable groups. Suppose $H$ acts on $N$ as a group of automorphisms such that the map $(n, h) \rightarrow h(n)$ from $N \times H$ into $N$ is continuous. Then the product space $N \times H$ can be made into a locally compact second countable group by assigning the product topology and defining the multiplication operation as

$$
\left(n_{1}, h_{1}\right) o\left(n_{2}, h_{2}\right)=\left(n_{1} h_{1}\left(n_{2}\right), h_{1} h_{2}\right), \quad n_{1}, n_{2} \in N, h_{1}, h_{2} \in H .
$$

The group obtained in this manner is called the semidirect product of $N$ and $H$ and is denoted by $N \odot H$.

Remark. Note that $N$ and $H$ can be considered as subgroups of $N \odot H$ in a natural manner. In such a case $N$ is a closed normal subgroup and $H$ is a closed subgroup.

The space $\mathfrak{R}^{4}$ can be identified with the space of all $2 \times 2$ Hermitian matrices through the map

$$
\tau: \boldsymbol{x}=\left(x_{0}, x_{1}, x_{2}, x_{3}\right) \rightarrow\left(\begin{array}{ll}
x_{0}+x_{1} & x_{2}+i x_{3} \\
x_{2}-i x_{3} & x_{0}-x_{1}
\end{array}\right) .
$$

Any $h \in S L(2, \mathfrak{C})$ induces an automorphism in the translation group $\mathfrak{R}^{4}$ as follows:

$$
h: x \rightarrow \tau^{-1}\left(h \tau(\boldsymbol{x}) h^{*}\right) .
$$

This is the well known covering map from $S L(2, \mathfrak{C})$ onto the proper Lorentz group. We shall denote by $S$ and $T$, the space and time reflections defined in $\mathfrak{R}^{4}$ as follows:

$$
\left.\begin{array}{l}
S:\left(x_{0}, x_{1}, x_{2}, x_{3}\right) \rightarrow\left(x_{0},-x_{1},-x_{2},-x_{3}\right), \\
T:\left(x_{0}, x_{1}, x_{2}, x_{3}\right) \rightarrow\left(-x_{0}, x_{1}, x_{2}, x_{3}\right) .
\end{array}\right\}
$$


Let $F$ be the finite group (consisting of four elements) generated by $S$ and T. $S L(2, \mathfrak{C})$ acts on $\mathfrak{R}^{4}$ as a group of automorphisms according to (5.1). $F$ acts on $S L(2, \mathfrak{C})$ and $\mathfrak{R}^{4} \odot S L(2, \mathfrak{C})$ as follows:

$$
\begin{array}{r}
\left.\begin{array}{r}
S: h \rightarrow h^{*-1} \\
T: h \rightarrow h^{*-1} \\
S T: h \rightarrow h
\end{array}\right\} \text { if } h \in S L(2, \mathfrak{C}), \\
\left.\begin{array}{r}
S:(\boldsymbol{x}, h) \rightarrow\left(S \boldsymbol{x}, h^{*-1}\right), \\
T:(\boldsymbol{x}, h) \rightarrow\left(T \boldsymbol{x}, h^{*-1}\right), \\
S T:(\boldsymbol{x}, h) \rightarrow(S T \boldsymbol{x}, h) .
\end{array}\right\} \text { if } \quad(\boldsymbol{x}, h) \in \mathfrak{R}^{4} \odot S L(2, \mathfrak{C})
\end{array}
$$

Thus one can form the semidirect products $\left[\mathfrak{R}^{4} \odot S L(2, \mathfrak{C})\right] \odot F$ and $S L(2, \mathfrak{C}) \odot F$. The group $S L(2, \mathfrak{C}) \odot F$ acts on $\mathfrak{R}^{4}$ as a group of automorphisms (by successive application). Hence one can also form $\mathfrak{R}^{4} \odot[\operatorname{SL}(2, \mathfrak{C}) \odot F]$. Then we have the following lemma.

Lemma 5.1. $\left[\mathfrak{R}^{4} \odot S L(2, \mathfrak{C})\right] \odot F=\mathfrak{R}^{4} \odot[S L(2, \mathfrak{C}) \odot F]$.

Proof. The proof is straightforward and left to the reader.

Definition 5.2. The group $\mathscr{P}=\mathfrak{R}^{4} \odot[S L(2, \mathfrak{C}) \odot F]$ is called the extended Poincaré group.

We shall now proceed to the description of all the multipliers of the extended Poincaré group. The connected component of the identity of $\mathscr{P}$ is $\mathscr{P}_{0}=\mathfrak{R}^{4} \odot S L(2, \mathfrak{C})$. It is a well known result of Bargmann and Whitehead (cf. [6] Theorem 5.5, page 34, and Corollary 1, page 51) that every multiplier of $\mathscr{P}_{0}$ is trivial. Since by Lemma $5.1 \mathscr{P}=\mathscr{P}_{0} \odot F$, any element $g \in \mathscr{P}$ can be uniquely written as $g^{0} a$ where $g^{0} \in \mathscr{P}_{0}$ and $a \in F$. An analysis similar to that of Mackey in the unitary situation (cf. Theorem 5.6, page 41 in [6]) and the fact that $\mathscr{P}_{0}$ has no nontrivial characters yields the following lemma.

Lemma 5.2. Let $\omega$ be any multiplier for $F$. Then the function $\sigma$ defined $b y$

$$
\sigma_{\omega}\left(g_{1}^{0} a_{1}, g_{2}^{0} a_{2}\right)=\omega\left(a_{1}, a_{2}\right) \quad \text { for all } g_{1}^{0}, g_{2}^{0} \in \mathscr{P}_{0}, \quad a_{1}, a_{2} \in F
$$

is a multiplier for $\mathscr{P}$. Conversely every multiplier $\sigma$ for the group is equivalent to a $\sigma_{\omega}$ for some multiplier $\omega$ of the group $\Gamma$.

Thus the problem of describing all the multipliers of $\mathscr{P}$ reduces to describing those of the finite group $F$. We recall from Definition 3.3 that every multiplier of $F$ is defined with respect to a subgroup $F^{+}$which has either two or four elements. Upto equivalence the multipliers of $F$ are described by the following lemma.

Lemma 5.3. Let $\Phi$ be a finite abelian group consisting of four elements $e, a, b, c$ such that $e$ is the identity, $a^{2}=b^{2}=c^{2}=e, a b=c, b c=a$ and $c a=b$. 
If $\Phi^{+}=\Phi$, then every multiplier with respect to $\Phi^{+}$is equivalent to the one which is identically equal to unity or the multiplier $\omega_{0}$ given by the following table:

Table (i)

\begin{tabular}{rrrrr} 
& $e$ & $a$ & $b$ & $c$ \\
\hline$e$ & 1 & 1 & 1 & 1 \\
$a$ & 1 & 1 & $i$ & $-i$ \\
$b$ & 1 & $-i$ & 1 & $i$ \\
$c$ & 1 & $i$ & $-i$ & 1
\end{tabular}

If $\Phi^{+}=\{e, a\}$, then every multiplier with respect to $\Phi^{+}$is equivalent to a multiplier $\omega_{\alpha \beta}^{a}$ given by the following table:

Table (ii)

\begin{tabular}{llllll} 
& $e$ & $a$ & $b$ & $c$ & \\
\hline$e$ & 1 & 1 & 1 & 1 & \\
$a$ & 1 & 1 & 1 & 1 & $\alpha= \pm 1, \beta= \pm 1$. \\
$b$ & 1 & $\alpha \beta$ & $\alpha$ & $\beta$ &
\end{tabular}

Proof. Since the proof is a straightforward algebra we leave it to the reader.

Remark. The extended Poincare group $\mathscr{P}$ has four distinct normal subgroups $\mathscr{P}^{+}$such that $\mathscr{P} / \mathscr{P}^{+}$has at the most two elements. They are given by

(1) $\mathscr{P}^{+}=\mathscr{P}$

(2) $\mathscr{P}^{+}=\left[\mathfrak{R}^{4} \odot S L(2, \mathfrak{C})\right] \odot F_{a}, a=S, T$ or $S T$

where $F_{a}$ denotes the subgroup of $F$ which contains the identity and the element $a$.

By Lemmas 5.2-5.3, there are two multipliers upto equivalence in the first case. One of them is identically equal to unity and other $\sigma_{0}$ is given by

$$
\sigma_{0}\left(g_{1}^{0} a_{1}, g_{2}^{0} a_{2}\right)=\omega_{0}\left(a_{1}, a_{2}\right) \text { for all } g_{1}^{0}, g_{2}^{0} \in \mathscr{P}_{0}, \quad a_{1}, a_{2} \in F
$$

where $\omega_{0}$ is determined by Table (i) of Lemma 5.3.

In the second case there are four multipliers $\sigma_{\alpha \beta}^{a}, \alpha= \pm 1, \beta= \pm 1$, given by

$$
\sigma_{\alpha \beta}^{a}\left(g_{1}^{0} a_{1}, g_{2}^{0} a_{2}\right)=\omega_{\alpha \beta}^{a}\left(a_{1}, a_{2}\right) \text { for all } g_{1}^{0}, g_{2}^{0} \in \mathscr{P}_{0}, \quad a_{1}, a_{2} \in F
$$

where $\omega_{\alpha \beta}^{a}$ is determined by Table (ii) of Lemma 5.3. Since $a$ can be any one of $S, T$ and $S T$ we obtain twelve distinct multipliers in this case.

Thus, on the whole, there are fourteen inequivalent multipliers for the group $\mathscr{P}$. 
We shall now proceed to describe the irreducible multiplier representations of $\mathscr{P}$ by following the method of Mackey [2]. Let $G=S L(2, \mathfrak{C}) \odot F$. Then $\mathscr{P}=\mathfrak{R}^{4} \odot G$. The $G$ action in $\mathfrak{R}^{4}$ introduces a natural action in the character group $R^{4}$ of $\Re^{4}$. Under this action $R^{4}$ splits into various orbits. Let $O$ be a typical orbit and $H$ be the stability subgroup of some fixed point in $O$. The orbit $O$ can then be considered as the homogeneous space $G / H$ of left cosets. We shall denote an arbitrary point of $O$ by $\boldsymbol{p}=\left(p_{0}, p_{1}, p_{2}, p_{3}\right)$ and define the value of the character $\boldsymbol{p}$ at $\boldsymbol{x}=\left(x_{0}, x_{1}, x_{2}, x_{3}\right)$ as $\exp -i\left(p_{0} x_{0}-p_{1} x_{1}-p_{2} x_{2}-p_{3} x_{3}\right)$. Any point of $\mathscr{P}$ can be denoted by $(\boldsymbol{x}, g)$ where $\boldsymbol{x} \in \mathfrak{R}^{4}$ and $g \in G$. Let $\sigma$ be a multiplier of $\mathscr{P}$ satisfying $\sigma\left(\left(\boldsymbol{x}_{1}, g_{1}\right),\left(\boldsymbol{x}_{2}, g_{2}\right)\right)=\sigma\left(g_{1}, g_{2}\right)$ for all $\left(\boldsymbol{x}_{1}, g_{1}\right),\left(\boldsymbol{x}_{2}, g_{2}\right)$. Then $\sigma$ defines a multiplier for $G$. Suppose $\left\{\mathscr{H}, V_{g}, P(E)\right\}$ is an imprimitivity system for the group $G$ on $O$ where the representation $g \rightarrow V_{g}$ has the multiplier $\sigma$. Define the operator $U_{x, g}$ by

$$
U_{(\boldsymbol{x}, g)}=\left\{\int\left(\exp -i\left[p_{0} x_{0}-p_{1} x_{1}-p_{2} x_{2}-p_{3} x_{3}\right]\right) P(d \boldsymbol{p})\right\} V_{g} .
$$

Then $(\boldsymbol{x}, g) \rightarrow U_{(\boldsymbol{x}, g)}$ is a $\sigma$ representation of $\mathscr{P}$. This is irreducible if and only if the imprimitivity system $\left\{\mathscr{H}, V_{g}, P(E)\right\}$ is irreducible. Every multiplier representation of $\mathscr{P}$ is determined in this way upto equivalence.

Following the procedure outlined above we shall now classify the "positive mass" representations of $\mathscr{P}$. Define for every $m>0$ the set $O_{m} \subset R^{4}$ by

$$
O_{m}=\left\{\boldsymbol{p}: p_{0} \neq 0, p_{0}^{2}-p_{1}^{2}-p_{2}^{2}-p_{3}^{2}=m^{2}\right\} .
$$

$O_{m}$ is an orbit of the group $G$ in $R^{4}$. Indeed, it is the orbit generated by the point $(m, 0,0,0)$. The stability subgroup of the point $(m, 0,0,0)$ is generated by $S U_{2}$ and the space reflection $S$. In fact, it is the direct product of $S U_{2}$ and the space reflection $S$. In fact, it is the direct product of $S U_{2}$ and $F_{S}$ where $F_{S}$ is the group consisting of the identity and $S$ only.

The irreducible multiplier representations corresponding to the orbit $O_{m}$ are now determined by the irreducible imprimitivity systems for $G$ on $O_{m}$. According to Theorems 4.1 and 4.2 such systems are in turn determined by the irreducible multiplier representations of $S U_{2} \times F_{S}$.

We shall now describe the invariant measure in $O_{m}$ and a cross section map $\gamma$ from $O_{m}$ into $G$. To this end, define

$$
\begin{aligned}
& O_{m}^{+}=\left\{\boldsymbol{p}: \boldsymbol{p} \in O_{m}, p_{0}>0\right\}, \\
& O_{m}^{\sim}=\left\{\boldsymbol{p}: \boldsymbol{p} \in O_{m}, p_{0}<0\right\} .
\end{aligned}
$$

There exists a $G$ invariant measure $\mu$ on $O_{m}$ given by

$$
\begin{aligned}
d \mu(\boldsymbol{p}) & =\frac{d p_{1} d p_{2} d p_{3}}{p_{0}} \text { if } \quad \boldsymbol{p} \in O_{m}^{+}, \\
& =\frac{d p_{1} d p_{2} d p_{3}}{-p_{0}} \text { if } \quad \boldsymbol{p} \in O_{m}^{-} .
\end{aligned}
$$


For any $\boldsymbol{p} \in O_{m}$, define

$$
\tau^{\prime}(\boldsymbol{p})=\left(\begin{array}{ll}
p_{0}+p_{1} & p_{2}+i p_{3} \\
p_{2}-i p_{3} & p_{0}-p_{1}
\end{array}\right)
$$

If $\boldsymbol{p} \in O_{m}^{+}, \tau^{\prime}(\boldsymbol{p})$ is a positive definite Hermitian matrix. If $\boldsymbol{p} \in O_{m}^{-}, \tau^{\prime}(\boldsymbol{p})$ is negative definite. Since every positive definite matrix has a unique positive definite square root, we can define

$$
\gamma^{+}(\boldsymbol{p})=m^{-\frac{1}{2}}\left[\tau^{\prime}(\boldsymbol{p})\right]^{\frac{1}{2}} \quad \text { if } \quad \boldsymbol{p} \in O_{m}^{+} .
$$

Then $\gamma^{+}$is a mapping from $O_{m}^{+}$into $S L(2, \mathfrak{C})$. If $\boldsymbol{p} \in O_{m}^{-}$, then $T \boldsymbol{p} \in O_{m}^{+}$. Hence we can define

$$
\gamma^{-}(\boldsymbol{p})=T \gamma^{+}(T \boldsymbol{p}) \quad \text { if } \quad \boldsymbol{p} \in O_{m}^{-}
$$

where the product of $T$ and $\gamma^{+}(T \boldsymbol{p})$ is taken in the group $G$. Now we write

$$
\begin{array}{rlll}
\gamma(\boldsymbol{p}) & =\gamma^{+}(\boldsymbol{p}) & \text { if } & \boldsymbol{p} \in O_{m}^{+}, \\
& =\gamma^{-}(\boldsymbol{p}) & \text { if } & \boldsymbol{p} \in O_{m}^{-} .
\end{array}
$$

It is now easy to verify that $\gamma$ is a cross section map, i.e., $\gamma$ is a one one Borel map from $O_{m}$ into $G$ such that $\gamma(\boldsymbol{p})(m, 0,0,0)=\left(p_{0}, p_{1}, p_{2}, p_{3}\right)$.

Representations arrived at by formula (5.3) on the basis of the orbit $O_{m}$ are called representations with mass $m$. We shall now classify these according to the multipliers described in the Remark after Lemma 5.3 and the irreducible $\sigma$ representations $L$ of $H=S U_{2} \times F_{S}$.

Case 1. $\mathscr{P}^{+}=\mathscr{P}, \sigma \equiv 1$.

In this case $L$ is an irreducible unitary representation of $H$. In particular $L_{S}^{2}=I$ and

$$
L_{u} L_{S}=L_{S} L_{u}=L_{u S} \text { for all } u \in S U_{2} .
$$

Thus $L_{S}= \pm I$ and $u \rightarrow L_{u}$ is a spin $j$ representation of $S U_{2}$ where $j$ is either an integer or half integer. For any spin $j$, the representations with $L_{S}=+I$ and $L_{S}=-I$ are inequivalent.

Case 2. $\mathscr{P}^{+}=\mathscr{P}, \sigma=\sigma_{0}$.

Since $\sigma_{0}(S, S)=1$, and $L_{S}$ is unitary we have $L_{S}^{2}=I ; L_{u} L_{S}=L_{S} L_{u}$. Thus $L_{S}= \pm I$ and the required representation of $\mathscr{P}$ is given by (5.3) where the $\sigma$ representation $g \rightarrow V_{q}$ is induced by the $\sigma$ representation $L$.

Case 3. $\mathscr{P}^{+}=\left[\mathfrak{R}^{4} \odot S L(2, \mathfrak{C})\right] \odot F_{S}, \sigma=\sigma_{\alpha \beta}^{S}$.

In this case the stability subgroup $H$ is included in $\mathscr{P}^{+}$. In (5.3), the $\sigma$ representation $g \rightarrow V_{g}$ is determined according to Theorem 4.2. $L$ is determined by a spin $j$ representation for $S U_{2}$ and $L_{S}= \pm I$ if $\alpha=+1$ and $L_{S}= \pm i I$ if $\alpha=-1$.

Case $4 a \cdot \mathscr{P}^{+}=\left[\Re^{4} \odot S L(2, \mathfrak{C})\right] \odot F_{T}, \sigma=\sigma_{\alpha \beta}^{T}, \alpha=+1$.

In this case $H \cap \mathscr{P}^{-} \neq \theta$. In (5.3), the $\sigma$ representation $g \rightarrow V_{g}$ is induced by a $\sigma$ representation $L$ of $H$ where $L_{S}$ is antiunitary. Since 
$\sigma(S, S)=1, L_{S}^{2}=I$. By Lemma $2.2, L_{S}$ is a conjugation. Since $L_{u} L_{S}=L_{S} L_{u}$ for all $u \in S U_{2}, u \rightarrow L_{u}$ has a real character. Hence the spin must be an integer. $L_{S}$ can now be chosen to be the complex conjugation in the basis in which the matrix entries of $u \rightarrow L_{u}$ are real.

Case $4 b . \mathscr{P}^{+}=\left[\mathfrak{R}^{4} \odot S L(2, \mathfrak{C})\right] \odot F_{T}, \sigma=\sigma_{\alpha \beta}^{T}, \alpha=-1$.

In (5.3), the $\sigma$ representation $g \rightarrow V_{g}$ is induced by a $\sigma$ representation $L$ of $H$ where $L_{S}$ is antiunitary. Further

$$
L_{\mathrm{S}}^{2}=-I, \quad L_{S} L_{u}=L_{u} L_{S}, \quad u \in S U_{2}
$$

Suppose $u \rightarrow L_{u}$ is a spin $j$ representation for $S U_{2}$. If $j$ is an integer there is a vector $v$ such that $L_{u} v=v$ for all $u$ in a one parameter subgroup $K$ of $S U_{2}$.

If $\boldsymbol{v}^{\prime}$ is another vector such that $L_{u} \boldsymbol{v}^{\prime}=\boldsymbol{v}^{\prime}$ for all $u \in K$, then $\boldsymbol{v}^{\prime}$ is a constant multiple of $\boldsymbol{v}$. Hence $L_{S} v=c \boldsymbol{v}$ where $c$ is a scalar of modulus unity. We have

$$
-\boldsymbol{v}=L_{S}^{2} \boldsymbol{v}=L_{S}(c \boldsymbol{v})=\bar{c} L_{S} \boldsymbol{v}=\boldsymbol{v} .
$$

Hence $v=0$. This implies that $j$ cannot be an integer. It is now easy to show that in a weight basis, $L_{S}$ is given by

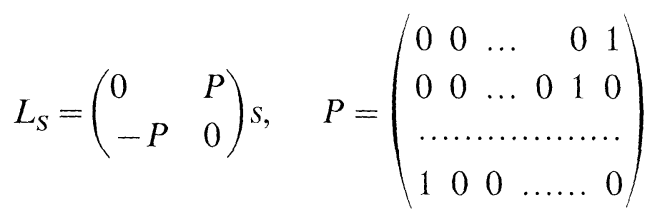

where $P$ is a $j+\frac{1}{2} \times j+\frac{1}{2}$ matrix and $s$ is complex conjugation in the chosen basis.

Cases $5 a$ and $5 b \cdot \mathscr{P}^{+}=\left[\mathfrak{R}^{4} \odot S L(2, \mathfrak{C})\right] \odot F_{S T}, \sigma=\sigma_{\alpha \beta}^{S T}, \alpha= \pm 1$.

The representations are determined exactly as in cases $4 \mathrm{a}$ and $4 \mathrm{~b}$. The only difference is in replacing $T$ by $S T$.

\section{References}

1. Foldy, L.: Synthesis of covariant particle equations. Phys. Rev. 102, 568-581 (1956).

2. Mackey, G. W.: Unitary representations of group extensions I. Acta Math. 99, 265-311 (1958).

3. - Mathematical foundations of quantum mechanics. New York: Benjamin 1963.

4. - Group representations. Mimeographed notes of lectures delivered at the Oxford Mathematics Institute 1966-67.

5. Parthasarathy, K. R.: Probability measures on metric spaces. New York: Academic Press 1967.

6. - Multipliers on locally compact groups, Vol. 93, Lecture Notes in Mathematics. Berlin-Heidelberg-New York: Springer 1969. 
7. Streater, R. F., Wightman, A. S.: PCT, spin, statistics, and all that. New York: Benjamin 1964.

8. Varadarajan, V. S.: Geometry of quantum theory, Vol. I. Princeton: Van Nostrand 1968.

9. Von Neumann, J.: Mathematical foundations of quantum mechanics. Princeton: University Press 1955 (English translation).

10. Wigner, E. P.: On the unitary representations of the inhomogeneous Lorentz group. Ann. Math. 40, 149-204 (1939).

\author{
K. R. Parthasarathy \\ University of Manchester \\ Statistical Laboratory \\ Department of Mathematics \\ Manchester, Great Britain
}

